\title{
Wie der Beweis der Vermutung von Baudet gefunden wurde
}

Bartel Leendert van der Waerden

Artin, Schreier und ich gingen im Jahr 1926 öfter im Curiohaus in Hamburg essen und unterhielten uns dabei über mathematische und andere Fragen. Einmal erzählte ich ihnen über eine Vermutung des früh verstorbenen holländischen Mathematikers Baudet. Sie lautete:

Teilt man die Gesamtheit der natürlichen Zahlen 1,2,3,.. in zwei Klassen ein, so enthält mindestens eine dieser Klassen eine arithmetische Progression von l Gliedern, wobei 1 eine beliebig grosse vorgegebene Zahl ist.

Nach dem Essen gingen wir in Artins Zimmer im damaligen Mathematischen Institut an der Rothenbaumchaussee und überlegten uns gemeinsam vor der Wandtafel an Hand von kleinen Kreidezeichnungen wie man wohl die Vermutung beweisen könnte. Wir stellten allerlei Überlegungen an und hatten ein paar Einfälle, die der Überlegung eine neue Richtung gaben und schliesslich zur Lösung führten.

Die Psychologie des Findens in der Mathematik ist eine schwierige Sache. Die meisten Mathematiker publizieren nur ihre Endergebnisse mit möglichst kurzen Beweisen, aber sie verraten uns nicht, wie sie darauf gekommen sind. Auch erinnern sie sich nachträglich

Wir drucken hier einen Beitrag von B.L. van der Waerden ab, der in Abh. Math. Sem. Univ. Hamb. 28 (1965), 6-15 erschienen ist. Eine davon nur geringfügig abweichende Version wurde bereits vorher in El. Math. 9 (1954), 49-56 veröffentlicht und von dort in ein Sammelheft Einfall und Überlegung übernommen, das 1954 beim Birkhäuser Verlag erschienen ist (Nachdruck 1968, 1973). Wir haben uns hier für die erstgenannte Version entschieden, weil sie offensichtlich das nur wenig überarbeitete Vortragsmanuskript reproduziert. So lassen sich hier auch charakteristische Eigenheiten des Vortragsstils von van der Waerden erkennen. - Wir danken der Schriftleitung der Abhandlungen des Mathematischen Seminars der Universität Hamburg für die Erlaubnis zu diesem Nachdruck. ust

*) Als Vortrag gehalten in der Universität Hamburg auf einer Gedenkfeier anlässlich des Todestages von Emil Artin am 19. Dezember 1963. 
nicht an alles, was ihnen durch den Kopf gegangen ist. Es fällt uns schwer, die eigenen vorbereitenden Überlegungen so wiederzugeben, dass auch andere sie verstehen. Die kurzen Andeutungen, in denen man mit sich selbst spricht, lassen sich ohne Präzisierung und Erläuterung nicht mitteilen, und durch die Präzisierung werden die Gedanken geändert.

Im Fall unseres Gespräches über die Vermutung von Baudet liegen aber die Bedingungen für die Wiedergabe viel günstiger. Denn alle Gedanken, die sich bei uns bildeten, wurden sofort ausgesprochen und durch Zeichnungen an der Tafel verdeutlicht. Wir veranschaulichten die Zahlen 1,2,3,.. der beiden Klassen durch äquidistante Kreidestriche auf zwei parallelen Geraden. Was ausgesprochen und gezeichnet wird, kann man viel besser festhalten und reproduzieren als blosse Gedanken. Also ein idealer Fall, um den Prozess des Findens zu analysieren, soweit er sich im bewussten Denken abspielt, und die bewussten Überlegungen abzugrenzen gegen die mysteriösen "Einfälle", die uns manchmal plötzlich ins Bewusstsein treten.

Wenn nur eine zweigliedrige Progression verlangt wird $(l=2)$, so braucht man nicht alle Zahlen $1,2, \ldots$ zu betrachten, sondern es genügt, sich auf die Zahlen 1,2,3 zu beschränken. Wenn diese auf zwei Klassen verteilt werden, so müssen zwei zur gleichen Klasse gehören. Das ist klar.

Auch im Fall $l=3$ braucht man nicht alle Zahlen zu betrachten, sondern man kann sich auf die Zahlen von 1 bis 9 beschränken. Teilt man diese in zwei Klassen ein, so liegt in einer dieser Klassen stets eine dreigliedrige arithmetische Progression $a, a+b, a+2 b$, wie man durch Aufzählen der möglichen Fälle leicht zeigt. Die Zahlen von 1 bis 8 kann man wohl in zwei Klassen teilen, ohne dass man eine dreigliedrige Progression erhält, z.B. so: 1, 2, 5, 6 in der ersten, 3, 4, 7, 8 in der zweiten Klasse. Die Zahl 9 gerät dann aber in eine Zwangslage. Steckt man sie in die erste Klasse, so hat man die Progression 159, andernfalls die Progression 789. Ähnlich in allen anderen Fällen. Das hatte ich mir schon vor der Zusammenkunft mit Artin und Schreier überlegt.

Schreier stellte nun die Frage, ob die Vermutung von Baudet ganz allgemein (wie in den Fällen $l=2$ und $l=3$ ) sich dahin verschärfen liesse, dass immer nur ein endlicher Abschnitt der Zahlenreihe in Betracht gezogen werden muss, mit anderen Worten, ob es eine Schranke $N=N(l)$ gibt, so dass bereits bei der Einteilung der Zahlen von 1 bis $N$ in zwei Klassen eine von diesen Klassen eine l-gliedrige Progression enthält. Die Frage war nicht schwer zu beantworten. Wenn die Vermutung von Baudet überhaupt richtig ist, so überlegten wir uns, dann gibt es auch ein solches $N$. Eine bekannte mengentheoretische Sohlussweise, das "Diagonalverfahren", führt zu diesem Ergebnis. Man schliesst etwa so:

Gesetzt, es gäbe kein solches $N$, dann würde es für jedes $N$ eine Klasseneinteilung $E_{N}$ der Zahlen von 1 bis $N$ geben, in der keine Klasse eine l-gliedrige Progression enthält. Es gäbe also eine Folge $E_{1}, E_{2}, \ldots$ von solchen Klasseneinteilungen. Die Zahl 1 liegt bei allen diesen Einteilungen in einer der beiden Klassen. Also muss sie unendlich oft in der gleichen (ersten oder zweiten) Klasse liegen. Es gibt also eine unendliche Teilfolge $E_{1}^{\prime}, E_{2}^{\prime}, \ldots$ von Klasseneinteilungen, bei denen die Zahl 1 immer in der gleichen, etwa in der $i_{1}$-ten Klasse liegt. 
In den Einteilungen $E_{2}^{\prime}, E_{3}^{\prime}, \ldots$ hat auch die Zahl 2 ihren Platz in der ersten oder zweiten Klasse. Also muss es eine unendliche Teilfolge $E_{2}^{\prime \prime}, E_{3}^{\prime \prime}, \ldots$ geben, bei denen 2 immer in der gleichen, etwa in der $i_{2}$-ten Klasse liegt.

So weiter schliessend, findet man für jedes $n$ eine Teilfolge $E_{n}^{(n)}, E_{n+1}^{(n+1)}, \ldots$ von Klasseneinteilungen, in denen die Zahlen $1,2, \ldots, n$ alle in den gleichen Klassen liegen, nämlich 1 in der $i_{1}$-ten, 2 in der $i_{2}$-ten, $\ldots, n$ in der $i_{n}$-ten.

Nun bildet man eine "Diagonal-Klasseneinteilung" $E$ der natürlichen Zahlen 1,2,.., bei der 1 in der $i_{1}$-ten, 2 in der $i_{2}$-ten Klasse liegt, usw. Die Zahl $n$ liegt bei dieser Klasseneinteilung in der gleichen Klasse wie in der Einteilung $E_{n}^{(n)}$. Daher der Name Diagonalverfahren.

In der Einteilung $E$ würde es unter den gemachten Annahmen keine $l$-gliedrige arithmetische Progression geben, deren Glieder alle derselben Klasse angehören. Wenn es sie gäbe, würde nämlich eine der Einteilungen $E_{n}^{(n)}$ bereits eine solche Progression enthalten, entgegen der gemachten Annahme. So kommen wir zu einem Widerspruch, also muss die gemachte Annahme falsch gewesen sein.

Auf Grund dieser Bemerkung von Schreier versuchten wir nun, den Satz in der verschärften Form mit der Schranke $N(l)$ zu beweisen. Da die Fälle $l=2$ und $l=3$ schon erledigt waren, so konnten wir versuchen, einen Schluss von $l-1$ auf $l$ durchzuführen. Artin bemerkte dazu, dass die finite Verschärfung für die vollständige Induktion nur von Vorteil sein kann. Wenn man für $l-1$ die Existenz einer Schranke $N(l-1)$ voraussetzen kann, so hat man mehr Möglichkeiten, für $l$ etwas zu beweisen.

Artin machte sodann die Bemerkung, dass die Vermutung, wenn sie für zwei Klassen allgemein richtig ist, auch für $k$ Klassen gelten muss. Es sei zum Beispiel $k=4$. Dann kann man die Klassen zunächst zu zwei und zwei zusammennehmen. So erhält man eine gröbere Einteilung in nur zwei Klassen. In einer dieser beiden muss eine arithmetische Progression von $N(l)$ Gliedern liegen. Die Glieder dieser Progression kann man von $l$ bis $N(l)$ numerieren. Diese Nummern erscheinen nun wieder in zwei Klassen der feineren Klasseneinteilung eingeteilt, und nach dem Satz, den wir für zwei Klassen als richtig angenommen haben, muss in einer dieser Klassen eine Progression von $l$ Gliedern liegen.

So kommt man von zwei auf vier Klassen, genau so von vier auf acht Klassen usw. Die Klassenzahl kann also beliebig gross sein.

Wir versuchten nun, den Satz durch vollständige Induktion nach $l$ zu beweisen. Für $l=2$ hat man das sogenannte "Schubfachprinzip": Wenn $k+1$ Dinge auf $k$ Schubfächer verteilt werden, so muss eines der Fächer mindestens zwei Dinge enthalten. Ein sehr nützliches Prinzip, das Dirichlet in der Zahlentheorie mit Erfolg angewandt hat.

Artin erwartete - und der Erfolg hat ihm recht gegeben -, dass die Verallgemeinerung von zwei auf $k$ Klassen für die Induktion von Vorteil sein würde. Man kann nämlich, so meinte er, nun versuchen, die Vermutung für ein beliebiges $k$ und für die Länge $l$ zu beweisen, unter der Induktionsvoraussetzung, dass sie für alle $k$ und für die Länge $l-1$ schon bewiesen sei.

Diese zunächst etwas unbestimmte Überlegung wurde im weiteren Verlauf der Diskussion, in der Hauptsache durch Artin, folgendermassen verschärft. Es soll etwa die Vermutung für zwei Klassen und für Progressionen der Länge $l$ bewiesen werden. Sind 
alle ganzen Zahlen in zwei Klassen eingeteilt, so sind zum Beispiel die Tripel aufeinanderfolgender Zahlen automatisch in acht Klassen eingeteilt, denn die drei Zahlen des Tripels können unabhängig voneinander in Klasse 1 oder 2 liegen, und das gibt $2^{3}=8$ Möglichkeiten. Man kann nun diese Zahlentripel durchnumerieren, etwa indem man jeweils die Anfangszahl des Tripels als Nummer nimmt, so dass Tripel Nummer $n$ aus den Zahlen $n, n+1$ und $n+2$ besteht. Die Nummern erscheinen dann in acht Klassen eingeteilt, und auf diese acht Klassen kann man unbedenklich die Induktionsvoraussetzung anwenden.

Dasselbe gilt, wenn man "Blöcke" von mehr als drei aufeinanderfolgenden Zahlen betrachtet. Nach der Induktionsvoraussetzung gibt es unter genügend vielen aufeinanderfolgenden Blöcken eine $(l-1)$-gliedrige arithmetische Progression von Blöcken. Das "Muster" der Verteilung der Zahlen auf die Klassen, das wir in einem dieser Blöcke vorfinden, wiederholt sich genau so in allen $l-1$ Blöcken der Progression. Vielleicht, so meinte Artin, geben diese sich wiederholenden Muster uns die Mittel zur Konstruktion einer $l$-gliedrigen Folge. Ausserdem enthalten die Blöcke selbst, wenn sie genügend lang sind, $(l-1)$-gliedrige arithmetische Progressionen von Zahlen einer Klasse. Auch diese können zur Konstruktion benutzt werden.

Wir versuchten nun, da im Fall $l=2$ der Satz sicher richtig ist, von $l=2$ auf $l=3 \mathrm{zu}$ schliessen, und zwar nahmen wir zunächst zwei Klassen an (ohne Rücksicht darauf, dass dieser Fall schon vorher durch direkte Aufzählung aller Fälle erledigt war). Wir zeichneten die Zahlen als kleine Querstriche im waagrechten Abstand 1 auf zwei waagrechten Linien, die die beiden Klassen darstellen sollten.

Unter je drei aufeinanderfolgenden Zahlen muss es nach der Induktionsvoraussetzung, das heisst in diesem Fall nach dem Schubfachprinzip zwei geben, die derselben Klasse angehören, etwa der ersten. Setzen wir nun die arithmetische Progression, die mit diesen beiden Strichen anfängt, fort, so können wir annehmen, dass der dritte Strich nicht mehr der ersten Klasse angehört (sonst wären wir ja schon fertig), sondern der zweiten. Somit ergibt sich das Bild der Figur 1. Soweit wurden alle Überlegungen von uns gemeinsam angestellt. Ich überlegte mir nun weiter folgendes.

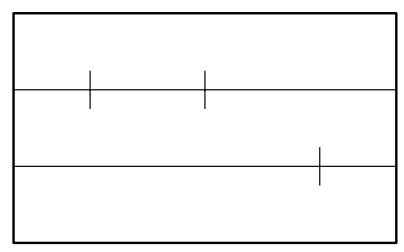

Fig. 1

In jedem Block von fünf aufeinanderfolgenden Zahlen muss ein Muster von der Art der Figur 1 vorkommen, denn unter den ersten drei Zahlen des Blockes muss es schon zwei geben, die derselben Klasse angehören und diese zweigliedrige Progression kann dann innerhalb des Blockes zu einer dreigliedrigen Progression fortgesetzt werden. 
Solche Muster wiederholen sich. Denn die Blöcke von fünf Zahlen sind ja in $2^{5}=32$ Klassen eingeteilt, und unter 33 aufeinanderfolgenden Blöcken ${ }^{1)}$ muss es nach dem Schubfachprinzip mindestens zwei gleiche geben. So ergibt sich das Bild der Figur 2, wobei der waagrechte Abstand zwischen dem Anfang des ersten und des zweiten Blockes höchstens 32 beträgt.

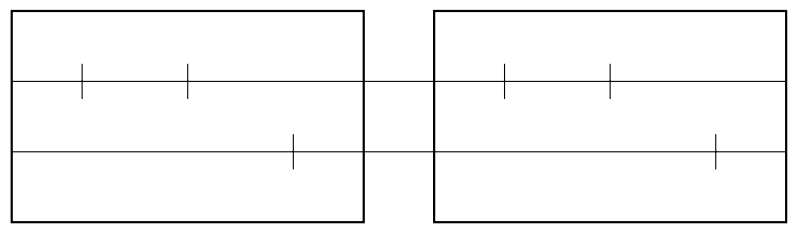

Fig. 2

Das gibt aber immer noch keine dreigliedrige Progression. Um eine solche zu erhalten, habe ich den zweiten Block von fünf Zahlen noch einmal um dieselbe Strecke verschoben und die dreigliedrige Progression betrachtet, die aus den angestrichenen Zahlen durch diese Verschiebung entsteht.

Die dritte Zahl dieser verschobenen Progression hat nun keinen Ausweg mehr. Entweder sie gehört in die erste Klasse: dann gibt es dort die arithmetische Progression $a, a, a$, oder sie gehört in die zweite Klasse: dann gibt es die Progression $b, b, b$ in der zweiten Klasse (Fig. 3).

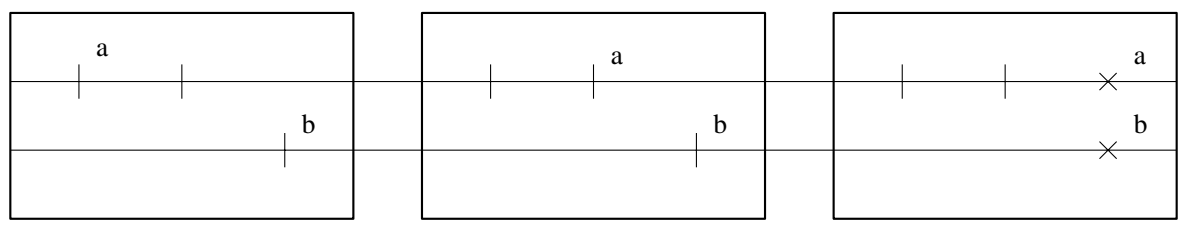

Fig. 3

Dieser Beweis galt zunächst nur für den bereits früher erledigten Fall $k=2, l=3$. Trotzdem hatte ich, als ich ihn vorbrachte, das sichere Gefühl, nun den allgemeinen Beweis in Händen zu haben.

Artin und Schreier glaubten es noch nicht. Da führte ich ihnen den analogen Beweis für den nächst höheren Fall $k=3, l=3$ vor.

In diesem Fall kann man zunächst genau dieselbe Überlegung anstellen (mit Blöcken zu sieben statt zu fünf und mit Abstand $3^{7}$ statt $2^{5}$ ), aber nun hat die dritte Zahl im dritten Block wohl einen Ausweg. Sie kann in die dritte Klasse hinein, und man erhält das in Figur 4 dargestellte Muster.

In jedem grossen Block von $3^{7}+3^{7}+7=h$ aufeinanderfolgenden Zahlen gibt es ein solches Muster. Nun zerfallen die grossen Blöcke in $3^{h}$ Klassen. Unter je $3 h+1$

1) "Aufeinanderfolgend" soll heissen: jeweils um Eins verschoben. 


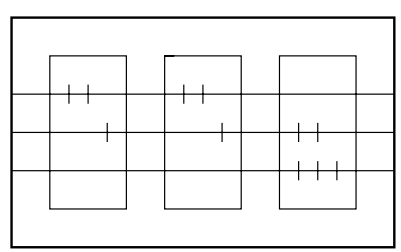

Fig. 4

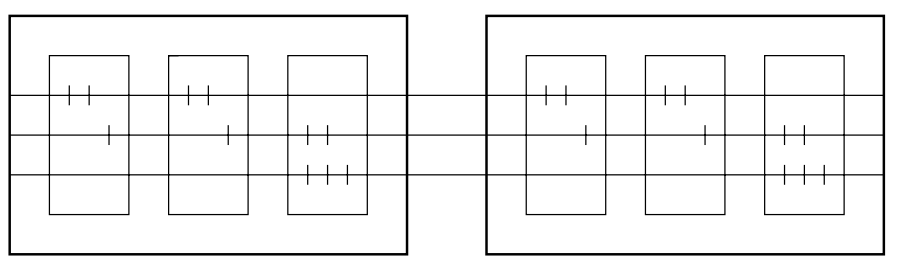

Fig. 5

aufeinanderfolgenden grossen Blöcken gibt es nach dem Schubfachprinzip zwei gleiche. In diese zeichne man die kleinen Blöcke hinein, und man erhält das Bild der Figur 5.

Jetzt verschiebe man den grossen Block, und man erhält dann an der Stelle der verschobenen Zahl $c$ entweder eine Progression $a, a, a$ in der ersten Klasse oder eine Progression $b, b, b$ in der zweiten oder $c, c, c$ in der dritten (Figur 6).

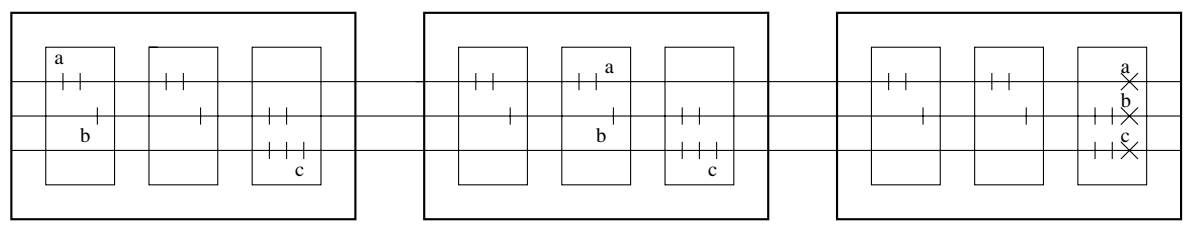

Fig. 6

Jetzt war es allen Beteiligten klar, dass für $l=3$ das Beweisverfahren sich auf beliebige $k$ übertragen lässt. Aber Artin und Schreier wollten nun noch den Fall $l=4$ sehen.

Ich nahm zunächst wieder zwei Klassen an. Nach dem bereits Bewiesenen gibt es unter genügend vielen, sagen wir $n$ aufeinanderfolgenden Zahlen eine dreigliedrige Progression, deren Terme alle einer Klasse angehören. Setzt man die Progression fort, so wird der vierte Term der anderen Klasse angehören (sonst wären wir ja schon fertig). Alle vier Zahlen gehören einem Block von $g$ aufeinanderfolgenden Zahlen an, wobei $g$ das grösste Ganze aus

$$
n+\frac{n-1}{2}
$$

ist. In jedem solchen Block kommt also das in Figur 7 abgebildete Muster vor. 


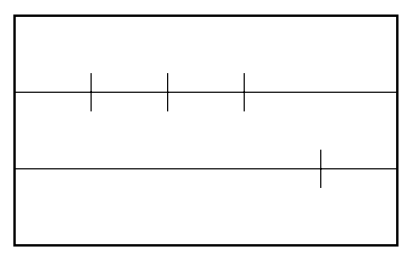

Fig. 7

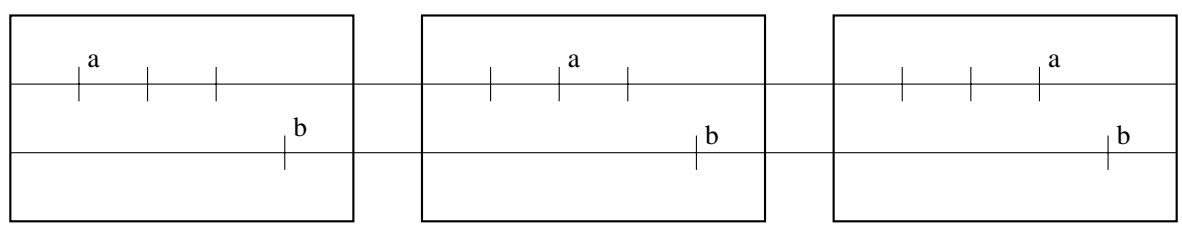

Fig. 8

Die Blöcke von der Länge $g$ sind in $2^{g}$ Klassen eingeteilt. Unter genügend vielen, sagen wir $n\left(3,2^{g}\right)$ aufeinanderfolgenden Blöcken gibt es also drei Blöcke in arithmetischer Progression, die derselben Klasse angehören. Das Muster im ersten Block wiederholt sich genau so im zweiten und dritten (Figur 8).

Fügt man nun noch einen vierten Block hinzu, so erhält man wieder zwangsläufig eine arithmetische Progression $a, a, a, a$ oder $b, b, b, b$.

Nachdem ich das ausgeführt hatte, war es uns allen dreien klar, dass es genau so weitergeht, dass man auch für beliebige $l$ nacheinander alle Fälle $k=2,3, \ldots$ erledigen und so den Schluss von $l-1$ auf $l$ allgemein vollziehen kann.

Der Beweis, den ich im "Nieuw Archief voor Wiskunde" 15, 212 (1927) dargestellt habe, ist die genaue Ausführung des hier anschaulich erläuterten Gedankenganges. Dem vorhin gebildeten Ausdruck (1), der allgemein so lautet:

$$
n+\left[\frac{n-1}{l-2}\right]
$$

entspricht im "Nieuw Archief” beim Schluss von $l-1$ auf $l$ der Ausdruck

$$
n(l-1, k)+\left[\frac{n(l-1, k)-1}{l-2}\right] \text {. }
$$

Der Beweis, den Chintschin in seinem sehr schönen Büchlein ${ }^{2)}$ bringt, ist nicht wesentlich von meinem Beweis verschieden; nur nimmt Chintchin statt (2) einfach $2 n$. Weiter betrachtet er statt aufeinanderfolgender Blöcke solche, die nebeneinanderstehen und sich

2) A.J. Chintchin: Drei Perlen der Zahlentheorie (Russisch 1947; Deutsch 1951, Akademie-Verlag, Berlin; Englisch 1952). Einen kürzeren Beweis eines allgemeineren Satzes gab E. Witt in: Mathematische Nachrichten 6, S. 201 (1952). 
berühren, wie $(a, \ldots, a+b-1)$ und $(a+b, \ldots, a+2 b-1)$, wodurch die Abschätzungen etwas gröber werden.

Ich will nun versuchen, die von Artin, Schreier und mir angestellten Überlegungen etwas näher zu analysieren und zu untersuchen, an welchen Stellen neue Einfälle den bewussten Überlegungen eine neue Richtung gaben.

Am Anfang stand ein Einfall von Schreier. Er fragte: Wäre es nicht möglich, der Vermutung von Baudet eine finite Verschärfung zu geben, indem man sich auf einen endlichen Abschnitt der Zahlenreihe beschränkt? Die Frage lag auf der Hand, denn in den Beispielen $l=2$ und $l=3$ war die finite Verschärfung schon gegeben. Der Beweis auf Grund des Diagonalverfahrens, der oben wiedergegeben wurde, war für jeden von uns dreien eine reine Routine-Angelegenheit, da wir alle drei mit diesem Verfahren vertraut waren. Aber der Einfall von Schreier bestimmte die Richtung, die unsere Überlegungen jetzt nahmen.

Dass wir eine Induktion nach $l$ versuchen wollten, war nur natürlich. Dass die finite Verschärfung die Induktion erleichtern würde, war zu erwarten.

Der nächste wesentliche Schritt wurde von Artin gemacht. Er hatte den Einfall, den Satz von 2 auf $k$ Klassen zu verallgemeinern. Veranlasst wurde dieser Einfall wahrscheinlich durch die Überlegung, dass im Fall einer zweigliedrigen Progression die Verallgemeinerung auf $k$ Klassen evident richtig ist (Schubfachprinzip).

Der oben dargestellte Beweis, dass die Vermutung von Baudet, wenn sie für zwei Klassen richtig ist, auch für $k$ Klassen richtig sein muss, stammt von Artin. In diesem Beweis steckt eine Idee, die nachher in meinem Beweis der verschärften Vermutung von Baudet eine zentrale Rolle spielen sollte, nämlich: Wenn die Vermutung für den Abschnitt von 1 bis $N$ richtig ist, so ist sie auch für jede $N$-gliedrige arithmetische Progression richtig, da man die Glieder dieser Progression ja von 1 bis $N$ numerieren kann.

Bei einem Schluss von $l-1$ auf $l$ ist es immer vorteilhaft, wenn man für $l-1$ möglichst viel voraussetzen kann und für $l$ möglichst wenig zu beweisen sich vornimmt. Im Sinne dieser Überlegung nahmen wir für $l-1$ die Richtigkeit der Vermutung, also die Existenz von $N(l-1, k)$ für alle $k$ an und versuchten zunächst für den nächsthöheren Wert $l$ und für ein einziges $k$, z.B. für $k=2$, die Existenz von $N(l, k)$ zu beweisen. Um die Gedanken zu bestimmen, nahmen wir zunächst $l=3$ an, weil hier der genaue Wert

$$
N(l-1, k)=N(2, k)=k+1
$$

nach dem Schubfachprinzip bekannt war. Damit war der Plan des Beweises vorgezeichnet.

Wir betrachteten also zunächst den Fall $k=2, l=3$. Durch direkte Aufzählung aller Möglichkeiten war dieser Fall zwar früher schon erledigt, aber wir suchten einen Beweisgedanken, der sich vielleicht auf höhere Fälle übertragen lassen würde. "Immer mit den ganz einfachen Beispielen anfangen", pflegte Hilbert zu sagen.

Der nächste entscheidende Einfall stammte von Artin. Wir können die Induktionsvoraussetzung, so sagte Artin, nicht nur auf Zahlen, sondern auch auf Blöcke von aufeinanderfolgenden Zahlen anwenden; denn auch diese sind in Klassen eingeteilt. Die Anzahl der 
Klassen ist zwar grösser ( $k^{n}$ für Blöcke von $n$ aufeinanderfolgenden Zahlen), aber das schadet nichts, da wir die Induktionsvoraussetzung ja für alle $k$ zur Verfügung haben.

Durch den Einfall von Artin war die Wiederholbarkeit eines ganzen Blockmusters gesichert und wir konnten die Figur 2 zeichnen, die im ersten Block zwei Zahlen der ersten und eine der zweiten Klasse in arithmetischer Progression zeigt, im zweiten Block die entsprechenden Zahlen in denselben Klassen.

Die Figur 2 zeigt immer noch keine dreigliedrige Progression in einer Klasse. Wie könnte man sie erhalten? Wir schauten die Striche auf der Tafel einige Zeit schweigend an. Auf einmal hatte ich einen Einfall, begleitet von dem sicheren Gefühl: Das ist die Lösung. Ich verschob den zweiten Block der Fig. 2 noch einmal um dieselbe Strecke und erhielt so die Figur 3, in der die dritte Zahl des dritten Blockes in eine Zwangslage gerät, ähnlich wie die Zahl 9 in der früheren Überlegung in eine Zwangslage geraten war.

Der Einfall lag eigentlich ganz nahe. Die einzige Zahl in Fig. 3, die sowohl mit zwei Zahlen $a, a$ der ersten Klasse als mit zwei Zahlen $b, b$ der zweiten Klasse je eine arithmetische Progression $a, a, a$ oder $b, b, b$ bildet, ist die dort angekreuzte dritte Zahl des dritten Blockes.

Das Bemerkenswerteste an diesem Einfall war das Gefühl der vollkommenen Sicherheit, das ihn begleitete. Das gleiche Gefühl der Sicherheit hatte auch Poincaré als ihm beim Einsteigen in einen Omnibus plötzlich eine mathematische Idee einfiel, "ohne dass irgend etwas in seinen früheren Gedanken diese Idee vorbereitet hatte"3). Ich glaube, viele Mathematiker haben ähnliche Erinnerungen an plötzliche Einfälle, deren Herkunft sie nicht bestimmen können. Oft sind diese Einfälle von einem Gefühl der Sicherheit begleitet, das allerdings auch trügen kann.

Ich hatte also in diesem Fall die intuitive Überzeugung, dass genau dieselbe Beweismethode, die ich im Fall $k=2, l=3$ an der Tafel vorführte, auch in allen höheren Fällen zum Ziel führen würde.

Wie diese Überzeugung sich im Unbewussten bilden konnte, das weiss ich nicht. Ich glaube aber, erklären zu können, warum Artin und Schreier nicht so sicher waren, auch nachdem ich ihnen den Fall $k=2, l=3$ erklärt hatte. Sie sahen nur das Ergebnis: das Vorhandensein der Progression $a, a, a$ in der ersten oder $b, b, b$ in der zweiten Klasse. Ich aber hatte eine Methode gefunden, diese Progressionen zu bilden, und ich hatte das bestimmte Gefühl, dass diese Methode auch auf die höheren Fälle anwendbar sein würde.

Es ist, wie wenn einer Äpfel von einem Baum pflückt. Wenn man einen Apfel gepflückt hat und ein anderer hängt etwas höher, so kann es sein, dass man selbst weiss, dass man mit etwas mehr Anstrengung den andern Apfel auch noch erreichen kann, während ein Zuschauer, der nur sieht, dass man den einen Apfel gerade erreicht hat, darüber im Zweifel ist. Man hat eben nicht nur den Apfel, sondern auch das Gefühl der Bewegungen, die man ausgeführt hat, um ihn zu pflücken.

Das Gefühl, dass eine Beweismethode noch weiter reicht, ist manchmal trügerisch. Oft stellt sich nachher heraus, dass in den höheren Fällen eine neue Schwierigkeit auftaucht.

3) J. Hadamard: Psychology of Invention in the Mathematical Field. Siehe auch B.L. van der Waerden: Einfall und Überlegung; Birkhäuser, Basel 1954. 
Trotzdem gehören solche Ahnungen über die Tragweite von Beweismethoden zu den nützlichsten Wegweisern bei der mathematischen Forschung.

Jetzt habe ich Ihnen alles erzählt, was mir von jener denkwürdigen Stunde, in der wir drei gemeinsam eine "Perle" gefunden haben, noch in Erinnerung ist. Es war eine meiner schönsten Stunden.

Van der Waerden publizierte den Beweis der Vermutung von Baudet erstmals in Nieuw Arch. Wisk. 15 (1927), 212-216. In der Literatur wurde das Resultat in der Folge als Satz von van der Waerden angesprochen. Nach Richard Rado (siehe Math. Z. 36 (1933), 424-480; Proc. London Math. Soc. (II) 48 (1945), 122-160) und Walter Deuber (siehe Math. Z. 133 (1973), 109-123) lässt sich das Resultat in einen wesentlich allgemeineren Rahmen stellen. Rado betrachtete in seinen beiden Arbeiten homogene lineare Gleichungssysteme (mit rationalen Koeffizienten), welche die Eigenschaft haben, dass bei jeder Zerlegung der natürlichen Zahlen in endlich viele Klassen in mindestens einer Klasse eine Lösung existiert. Solche Gleichungssysteme, die er partitionsregulär nannte, konnte Rado auf erstaunlich einfache Weise charakterisieren und zeigen, dass sich die Aussage des Satzes von van der Waerden auf die Lösbarkeit eines partitionsregulären Gleichungssystems zurückführen lässt. Aus Rado's Charakterisierung partitionsregulärer Gleichungssysteme ergibt sich darüberhinaus das folgende überraschende Resultat: Bei einer Zerlegung der natürlichen Zahlen in endlich viele Klassen gibt es mindestens eine Klasse, in der jedes partitionsreguläre Gleichungssystem lösbar ist. Walter Deuber hat in seiner Dissertation unter der Leitung von Ernst Specker eine daran anschliessende Vermutung von Rado beweisen können: Es sei $S$ eine Teilmenge der natürlichen Zahlen, welche die Eigenschaft hat, dass in ihr jedes partitionsreguläre Gleichungssystem lösbar ist. Zerlegt man $S$ in endlich viele Klassen, so ist in mindestens einer dieser Klassen wiederum jedes partitionsreguläre Gleichungssystem lösbar. ust 